\title{
Lithium plating and stripping in the framework of a 3D electrochemical model
}

\author{
S. Hein ${ }^{\mathrm{a}, \mathrm{b}}$, and A. Latz ${ }^{\mathrm{a}, \mathrm{b}, \mathrm{c}}$ \\ ${ }^{a}$ Institute of Engineering Thermodynamics, German Aerospace Centre (DLR), 70569 \\ Stuttgart, Germany \\ ${ }^{\mathrm{b}}$ Helmholtz Institute Ulm for Electrochemical Energy Storage, 89081 Ulm, Germany \\ ${ }^{\mathrm{c}}$ Institute of Electrochemistry, University Ulm, 89069 Ulm, Germany
}

One of the major degradation processes in lithium-ion batteries is lithium plating. This mechanism describes the growth of a parasitic metallic lithium phase on the surface of the negative electrode. We present an extension to a 3D transport model, which allows for deposition and dissolution of an additional metallic lithium phase inside of a lithium-ion battery. The effect of lithium stripping and plating on the cell voltage can be investigated.

\section{Introduction}

There are several processes, which can lead to a lowering of the performance or reduction of the lifetime of lithium-ion batteries. These processes are commonly called degradation processes. One of these processes is the so called lithium plating. It is not possible to detected lithium plating directly in situ in a feasible way. One can see indirectly, that lithium plating happened via a plateau during discharge of a plated lithium-ion battery (1) (2). Figure 1 shows a sketch of this voltage plateau.

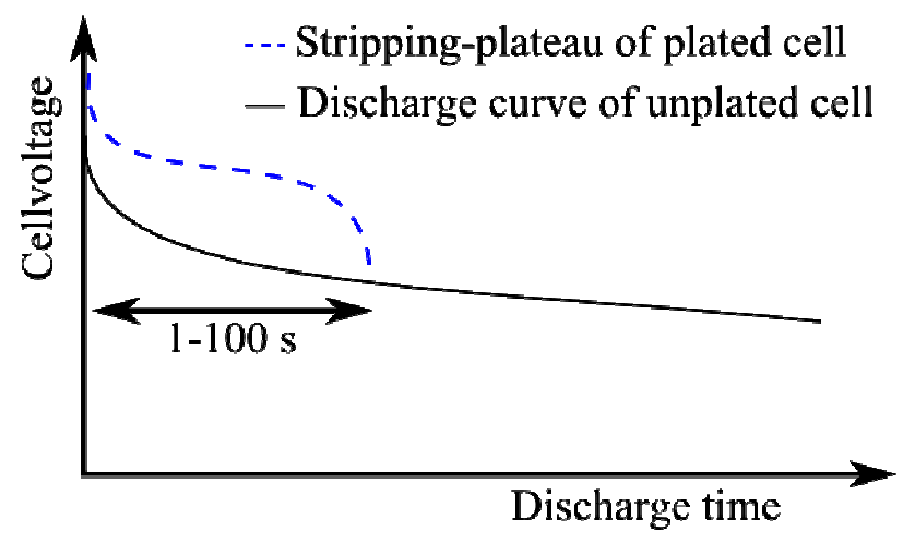

Figure 1. In this figure the cell voltage of a plated and unplated cell during discharge with a constant current is shown. During the discharge of a lithium-ion battery, which contains plated lithium, a plateau can be measured during the beginning. The height and length of this stripping plateau depends on the amount of plated lithium and the reaction constant of the plated lithium. 


\section{Lithium Plating}

Lithium plating is a degradation process, which happens at interface between the negative electrode and the electrolyte. The lithium plating reaction leads to the creation of a metallic lithium phase on top of the anode surface, mostly during the charge of a lithium-ion battery. Equation [1] shows the reaction of this step.

$$
\mathrm{Li}^{+} \text {Electrolyte }+\mathrm{e}_{\text {Solid }}^{-} \rightarrow \mathrm{Li}_{\text {Plated }}
$$

This deposition replaces the intercalation process, in which a lithium ion is transferred from the electrolyte into the host lattice of the negative electrode. While continuing the charging process of the battery, more and more lithium can accumulate on the surface of the anode. At the interface of this freshly created lithium metal, the degradation process of solid electrolyte interphase formation (SEI formation) can take place and therefor accelerate the loss of active lithium. Another risk of this metallic lithium phase is the occurrence of electrical short circuits, if the growth of the lithium reaches the opposing electrode (positive electrode).

The deposited lithium can also chemically intercalate into the supporting negative electrode (see equation [2]). This effect can be seen, if a battery cell, which contains plated lithium, is kept at rest (open circuit).

$$
\mathrm{Li}_{\text {Plated }} \rightarrow \mathrm{Li}_{\text {Solid }}
$$

\section{Model}

We present an extension to an existing electrochemical transportmodel (3) for lithiumion batteries. This extension allows for simulation of lithium plating and stripping inside of the microstructure of a lithium-ion battery based on intercalation materials. In the proposed extension, the process of lithium plating is modeled as a two-step process. Lithium is first adsorbed at the anode surface (Equation [3]), followed by insertion of these surface species into the host lattice (Equation [4]).

$$
\begin{aligned}
\mathrm{Li}^{+} \text {Electrolyte } & +\mathrm{e}_{\text {Solid }}^{-} \rightarrow \mathrm{Li}_{\text {Adsorped }} \\
\mathrm{Li}_{\text {Adsorped }} & \rightarrow \mathrm{Li}_{\text {Solid }}
\end{aligned}
$$

If the surface coverage reaches a critical limit, the adsorbed surface lithium nucleates into metallic lithium. These lithium nuclei start to cover the interface between the active material and the electrolyte. Additional deposition of lithium on this metallic surface layer will lead to growth of the metallic lithium phase into the electrolyte.

During discharge of a plated battery, the model allows for the shrinkage of the plated lithium film. This stripping part is followed by the dissolution of plated lithium on the surface, which allows lithium to be deintercalated directly from the active material. 


\section{Simulation}

The model described above is implemented in the framework of a 3D microstructure simulation tool (4). On this basis, the effect of microstructure, temperature and reaction kinetics on the onset and location of lithium plating can investigated. The first occurrence of plating in a lithium-ion battery can be confirmed to be at the interface between negative electrode and separator. In Figure 2 part of the microstructure of a lithium-ion battery and the resulting cell voltage for discharge with and without lithium is shown. Since the formation of a new SEI on the plated lithium is not yet included in the current model, the voltage drops to the value without plating once the whole lithium has been dissolved (as shown in Fig. 2.).
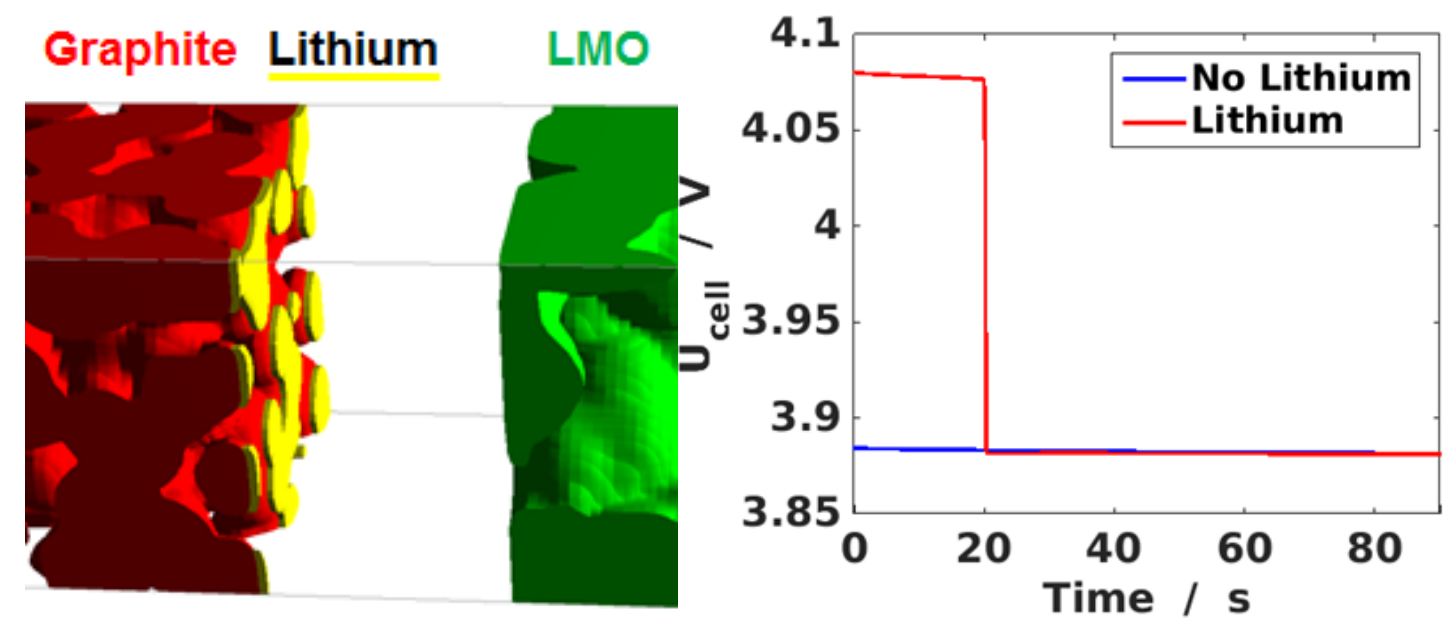

Figure 2. (left) Microstructure of a lithium-ion battery based on Graphite and LMO. At the interface between anode and separator a metallic lithium film is present. (right) During discharge the cell voltage of the battery with and without additional lithium phase is shown. A distinct voltage plateau for the case with lithium is visible.

\section{Acknowledgments}

The authors want to acknowledge the Bundesministerium für Bildung und Forschung for the financial support in the framework of BMBF Multibat 05M13PMA.

\section{References}

1. M. Petzl and M. A. Danzer, J. Power Sources, 254 (2014), pp. 80-87.

2. B.V. Ratnakumar, M.C. Smart, J. Electrochem. Soc. Trans. 25 (2010), pp. 241252.

3. A. Latz and J. Zausch, J. Power Sources, 196 (2011), pp. 3296-3302

4. BEST (Battery and Electrochemical Simulation Tool), http://www.itwm.fraunhofer.de/best 